\title{
Review of: "Pronominal anaphora resolution in Polish: Investigating online sentence interpretation using eye-tracking"
}

\author{
Carlos Gelormini-Lezama ${ }^{1}$
}

1 Universidad de San Andres

Potential competing interests: The author(s) declared that no potential competing interests exist.

This study investigates the resolution of anaphoric pronouns in Polish. Despite some limitations, this study makes a contribution by providing data from an understudied language in the field of refence processing. Although the results do not conclusively support any particular theory, they reinforce the need to view anaphoric processing as a complex phenomenon which involves syntactic, semantic and discourse factors.

The first experiment was designed as an offline survey to explore the preference of Polish readers with respect to the interpretation of sentences containing overt and null pronouns referring to subject or object antecedents. The second was an eye-tracking experiment in which the matching between pronouns (overt or null) and their antecedents (subject or object) was manipulated.

The survey showed that, overall, sentences with a null pronoun were interpreted as referring to subject antecedents whereas sentences with an overt pronoun were linked to object antecedents. The results from the eye tracking experiment showed that the violation of a preferred null pronoun-antecedent match did not generate an extra processing cost. Contrary to this, the overt pronoun was more sensitive to this kind of manipulation: there was an increase in the processing cost as the reader was forced to link the overt pronoun with an antecedent in subject position.

The initial claim that "the mechanism of anaphora resolution is subject to large cross-linguistic differences" is not really supported by previous research and it is also contradicted by this study. The whole point of an area of study devoted to the processing of anaphoric expression is based on the theoretically and empirically grounded assumption that there are universal mechanisms in human sentence processing in general, and in anaphoric processing in particular. Some variation with respect to these mechanisms is expected. Variation at all levels is the rule in language, rather than the exception. But, crucially, variation is constrained to a limited range of options. Therefore, it might be more accurate to say that the mechanisms of anaphora resolution are, contrary to this claim, quite similar across languages with some generally predictable and rule-governed variation. 
The article reviews some of the theories that try to capture generalizations about the process of anaphora resolution. However, it omits some relevant studies in Spanish, Italian and Portuguese and thus, it fails to offer a more balanced view of anaphoric resolution in null-subject languages. Incidentally, a minor terminological point should be considered as well: the term "pro-drop" is normally used to refer to languages in which it is possible to omit subjects or objects. Spanish is not a typical pro-drop language in this narrow sense because the omission of the subject is grammatical whereas the omission of the object is normally unacceptable. "Null subject" provides a better label for languages like Spanish and Italian.

One phenomenon that has been omitted in the literary review is the overt pronoun penalty (OPP) for which there is evidence in Spanish, Italian, Portuguese, among other languages, such that overt pronouns referring to subject antecedents generate a processing delay. Therefore, the claim that "an increased processing cost resulting from forcing a shift of reference from the syntactic subject of a sentence to the syntactic object is observed for Italian but not for Spanish" is, at the very least, controversial. The same holds for "in Italian, the interpretation of an overt pronoun that was forced to refer to a subject antecedent resulted in a significant processing penalty. This was not the case for native Spanish speakers, who could accommodate the less-preferred pronoun-antecedent match effortlessly." There is now plenty of evidence which strongly suggests that the processing of Italian and Spanish anaphoric expressions is more similar than previously thought.

In most experiments in anaphoric processing there is a main effect of antecedent salience, such that anaphors, in general, prefer subjects over objects. This is a main effect of antecedent salience and this is, in no way, "unexpected". Psycholinguistic research in different languages and with different methodologies has shown that sentences containing anaphors that refer to object antecedents are, overall, read more slowly than identical sentences containing anaphors that refer to subject antecedents. This effect, of course, may interact with other factors and it may not surface in certain very specific conditions. But in the kind of stimuli used in this study there was no reason to assume that this known effect would not be elicited.

In the eye-tracking experiment, the analysis of the critical area of interest suggests that the null pronoun is insensitive to the violation of the antecedent preference. This is a very interesting finding and it is different from what happens in Romance languages. The overt pronoun, on the other hand generates an increase in the processing cost. This pattern is a noteworthy result which should be further explored and discussed. The fact that the null pronoun is insensitive to the violation of antecedent preference is definitely an unexpected result which opens up questions for future research.

To sum up, this is a relevant study in the area of anaphoric processing, which highlights the importance of 
analyzing data from different languages. The findings are not earth shattering but they are consistent with previous research in other languages in that they suggest the need to consider a variety of factors involved in the process of pronoun resolution. Some minor but meaningful differences with previous research in other languages, especially with respect to the behavior of the overt pronoun in Polish and Romance languages, might make a relevant contribution to the expansion and elaboration of existing theories of anaphoric processing. 